\title{
Tuneable PTC effect in polymer-wax-carbon composite resistors
}

\author{
Thomas Maeder, Caroline Jacq, Ludivine Ammon \& Peter Ryser \\ Laboratoire de Production Microtechnique, École Polytechnique Fédérale de Lausanne (EPFL), CH-1015 \\ Lausanne, Switzerland \\ http://lpm.epfl.ch; thomas.maeder@epfl.ch \\ Version of record: Microelectronics International, 2014, in press
}

\begin{abstract}
:
- Purpose: The resistivity dependence on temperature of composite resistors made of carbon fillers dispersed in an organic matrix are known to be strongly affected by the matrix thermal expansion. High positive temperature coefficient (PTC) effects, i.e. essentially switching from resistive to quasi-insulating behaviour, can be caused by phase changes in the matrix and the assorted volume expansion, a behaviour that has been previously shown with both simple organic waxes and semicrystalline polymers. However, waxes become very liquid upon melting, possibly resulting in carbon sedimentation, and tuneability of semicrystalline polymers is limited.

- Approach: We therefore study a ternary polymer-wax-conductor (ethylcelluloseoctadecanol-graphite) composite resistor system, where polymer and wax fuse to a viscous liquid upon heating, and re-solidify and separate by crystallisation of the wax upon cooling.

- Findings: It is shown that with appropriate formulation, the resulting resistors exhibit strong PTC effects, linked with the melting and crystallisation of the wax component. The behaviour somewhat depends on sample history, and notably cooling speed.

- Limitations: The phase equilibria and transformation kinetics of the polymer-wax system (including possible wax polymorphism), as well as the exact mechanism of the conductivity transition, remain to be investigated.

- Originality/value: As many compatible polymer-wax systems with different melting/solidification behaviours are available, ternary polymer-wax-conductor composite PTC resistors allow a high tuneability of properties. Moreover, the high viscosity in the liquid state should largely avoid the sedimentation issues present with binary wax-conductor systems.
\end{abstract}

Keywords: composite resistors, carbon, polymer-wax matrix, PTC effect, resettable fuses Article Classification: research paper

\section{Introduction}

Besides high-reliability thick-film cermet resistors (Prudenziati and Dell'Acqua, 1994), composite resistive materials based on organic matrices (Strümpler and Glatz-Reichenbach, 1999), mainly on different forms of carbon (Chung, 2004), have many useful industrial properties and allow convenient low-temperature processing. Especially, for thick-film conductive and resistive materials, the use of organic resin matrices allows compatibility with 
a wide range of substrates, albeit at the cost of lower stability than their cermet analogues (Dziedzic et al., 1993; Serra et al., 2010).

One particularly interesting feature of such composite resistors (both in bulk and thick-film form) is the possibility of introducing a steep increase in resistivity above a given temperature: a "switching" positive temperature coefficient (PTC) effect, as opposed to the more linear one used for instance in platinum temperature sensors. This abrupt jump in resistivity is due to a $1^{\text {st }}$-order phase change in the matrix, i.e. mostly melting, as has been shown for model systems of exfoliated graphite or carbon nanotubes in hexadecane or even water (Zheng et al., 2011). However, such composites, whose matrix consists solely of small molecules, are not expected to be stable over many cycles, as the high fluidity of the molecular matrix in the molten state allows extensive particle rearrangements or even sedimentation.

Practical PTC composites applicable to resettable fuses (Doljack, 1981) and self-limiting heaters (Lukasik et al., 2003) are therefore based on polymers, which are highly viscous in the molten state. The main materials used are forms of high-density polyethylene (HDPE), which can achieve a quite high level of crystallinity and yield practical transition temperatures in the $120-140^{\circ} \mathrm{C}$ range (Heaney, 1996; Zhang et al., 2005; Chen et al., 2005), in line with the melting range of the crystallites. However, HDPE does not lend itself well to thick-film processing, and its transition temperature cannot be easily tuned.

In this work, we therefore explore a mixed approach (Łukasik et al., 2003; Maeder et al., 2012), akin to hot-melt adhesives, between a molecular (Zheng et al., 2011) and polymer matrix (Heaney, 1996; Zhang et al., 2005; Chen et al., 2005), whereby an amorphous polymer (ethylcellulose, EC) well-suited as a screen-printing vehicle is combined with a molecular crystalline wax (1-octadecanol, stearyl alcohol, C18E0):

- At high temperature, the wax melts and acts as a solvent for the matrix. However, due to the long-chain solvent and dissolved long-chain polymer, viscosity remains high and precludes sedimentation.

- Upon cooling, the wax crystallises out of the polymer, which solidifies both the now crystalline wax and the polymer, due to the now separate wax no longer acting as a plasticiser. Note that a very similar compound, 1-hexadecanol (cetanol) is indicated as a plasticiser for EC in supplier documentation (Hercules, 2002), but without mention of its observed tendency to crystallise out (Maeder, 2012 \& 2013).

Using this hot-melt matrix and graphite powder as a conductive phase, we expect a reversible jump in resistance somewhat below the melting point of the wax, $\sim 59^{\circ} \mathrm{C}$.

\section{Experimental}

\subsection{Materials and sample preparation}

Ethylcellulose (EC-4-48, 4 cps grade, 48\% hydroxyl) and octadecanol (C18E0) were purchased from Sigma-Aldrich, and synthetic near-spherical graphite (KS4, <4 $\mu \mathrm{m})$ was obtained from TIMCAL (Tab. 1). First, the matrix was prepared by dissolving EC-4-48 powder into liquid $\mathrm{C} 18 \mathrm{E} 0\left(\sim 100^{\circ} \mathrm{C}\right.$, under constant stirring), with a $1: 3$ mass ratio, which yields a waxy solid that is tougher than $\mathrm{C} 18 \mathrm{E} 0$ alone at room temperature. Various amounts of graphite were then included by hand mixing in the re-molten matrix.

For these preliminary experiments, the composites were not formulated in solvents for conventional screen printing. Instead, resistive strips were screen-printed directly $(\sim 1.5 \mathrm{~mm}$ width, $\sim 50 \mu \mathrm{m}$ thickness) in the hot state onto test patterns on alumina substrates, by hand through a metal mask, followed by uncontrolled cooling (estimated time for cooling below 
$30^{\circ} \mathrm{C} \sim 30 \mathrm{~s}$ ). Nevertheless, it is possible to formulate standard screen-printable pastes with such systems, either by keeping the wax dissolved in the solvent phase or by controlled crystallisation so that it becomes suspended in the vehicle together with the conductive filler (Maeder et al., 2012 \& 2013). Resistor lengths, as defined by the Ag electrodes (ESL 9912K, ElectroScience Laboratories, USA) ranged from 0.3 to $5.0 \mathrm{~mm}$ (Fig. 1).

Table 1. Used materials (density according to supplier data).

\begin{tabular}{|c|c|c|c|}
\hline Material & Code & Supplier & $\begin{array}{c}\text { Density } \\
{\left[\mathrm{kg} / \mathrm{m}^{3}\right]}\end{array}$ \\
\hline Ethylcellulose & EC / EC-4-48 & Sigma-Aldrich & $1^{\prime} 140$ \\
\hline 1-Octadecanol & C18E0 & Sigma-Aldrich & 812 \\
\hline Graphite, synthetic, $<4 \mu \mathrm{m}$ & KS4 & TIMCAL & $2 ' 255$ \\
\hline
\end{tabular}

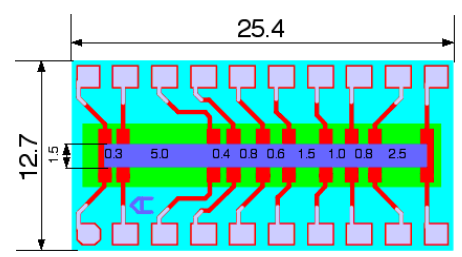

Figure 1. The used resistive test patterns (numbers on the resistive strip: effective resistor lengths, all dimensions in $\mathrm{mm}$ ).

\subsection{Resistance - temperature measurements}

The samples were tested in a custom-made system, controlled with two (for checking consistency) Pt1000 resistive temperature sensors, with 4-wire resistance being measured using a Keithley 2000 multimeter. Resistive test patterns were subjected to two heating / cooling cycles:

- A first temperature cycle was performed from $25^{\circ} \mathrm{C}$ to $80^{\circ} \mathrm{C}$, in regular $5 \mathrm{~K}$ steps.

- A second cycle was then carried out, starting at $25^{\circ} \mathrm{C}$, then $40^{\circ} \mathrm{C}$, then $47-58^{\circ} \mathrm{C}$ in $1 \mathrm{~K}$ steps, in order to examine the transition in detail.

Measurements were performed both during the heating and cooling phases ( 3 per temperature and altogether 18 resistors per composition). For each point, the temperature was stabilised for $\sim 3$ min before taking the measurement.

\subsection{Matrix viscosity measurements}

The apparent viscosity of the EC:C18E0 1:3 matrix was measured with a Mettler Rheomat R180 rheometer, during the cooling cycle, measuring temperature simultaneously. The measurement was carried out with combination 13 of the DIN 53019 measurement system, and applying a shear rate of $10 \mathrm{~s}^{-1}$. To obtain a slow cooling rate and better temperature uniformity, the bottom of the measurement end was inserted in water mantle of $\sim 1 \ell$ volume, which was progressively cooled $\left(\sim 10 \mathrm{~K} / \mathrm{h}\right.$ between 50 and $\left.45^{\circ} \mathrm{C}\right)$. 


\section{Results and discussions}

\subsection{General observations}

The evolution of the resistivity of the samples is shown in Fig. 2, for the first cycle, and Fig. 3 for the second. Altogether, it is apparent that the samples behave as switching-type PTC thermistors, i.e. essentially having a metal-insulator transition above a critical temperature somewhat below the melting point of the wax. All compositions roughly behave in a similar manner:

- During the heating phase, at low temperature, resistivity first increases smoothly with temperature, until the critical temperature is reached, somewhat below the melting point of the wax.

- Above this temperature, resistivity increases abruptly, and conductivity is essentially lost (resistance not measureable, resistivity $>10^{4} \Omega \cdot \mathrm{m}$ ).

- Upon cooling, conductivity is restored near the temperature it was lost.

- Resistivity then decreases smoothly with further cooling towards room temperature.

No observable effect of resistor length is found, which is expected, given the low-temperature preparation (no expected reaction with the electrode) and small expected microscopic length scale $(\sim 10 \mu \mathrm{m}$ vs. min. $300 \mu \mathrm{m}$ resistor length).

Comparing the different samples, resistivity is expectedly lower for higher filler loading. However, there is no observed effect, in the narrow studied composition range, of filler loading on switching temperature. Also, there is no apparent switching hysteresis. For the second, more accurate and slow cycle, all samples are conducting $\left(<10^{4} \Omega \cdot \mathrm{m}\right)$ at $55^{\circ} \mathrm{C}$, and insulating $\left(>10^{4} \Omega \cdot \mathrm{m}\right)$ at $57^{\circ} \mathrm{C}$, on both heating and cooling. Therefore, we conclude the transition temperature is $\sim 56^{\circ} \mathrm{C}$ in our system.

\subsection{Correlation with phase behaviour}

As expected, our samples, exhibit 'switching' PTC characteristics, with a switch temperature slightly lower than the melting point of the wax $\left(56\right.$ vs. $\left.\sim 59^{\circ} \mathrm{C}\right)$. An exact match is not expected, as the transformation is not simple melting of a pure phase, but the transformation of the bicomponent EC-C18E0 polymer-wax system. Assuming that the crystalline C18E0 phase does not dissolve any $\mathrm{EC}$, the reversible evolution of the matrix can therefore be written as:

$$
\text { EC (amorphous, with less dissolved } \mathrm{C} 18 \mathrm{E} 0)+\mathrm{C} 18 \mathrm{E} 0 \rightleftarrows \text { liquid }(\mathrm{EC}+\mathrm{C} 18 \mathrm{E} 0)
$$

As such, the transformation is not expected to occur at a single temperature, even at equilibrium, but rather over a temperature range. Nevertheless, as the overall 1:3 polymer:wax ratio is rather close to that of pure wax, it is not surprising that the observed transformation temperature is close to the wax melting point; although no data was found for the EC:C18E0 system, solubility studies of the higher 1-alkanols in many solvents (Domańska and Gonzalez, 1997; Hoerr et al., 1944) indicate, especially for the long alcohols such as C18E0, a rather flat liquidus curve near the pure alcohol. This also suggests a rather sharp - if not strictly at a single temperature - phase transformation behaviour, i.e. a strong increase of the crystalline wax phase upon further cooling below the temperature of its initial appearance. Also, the aforementioned studies indicate a low equilibrium solubility of $\mathrm{C} 18 \mathrm{E} 0$ at room temperature, with solubility at most (in 1-butanol) $<10 \%$ wt. at $20^{\circ} \mathrm{C}$. 

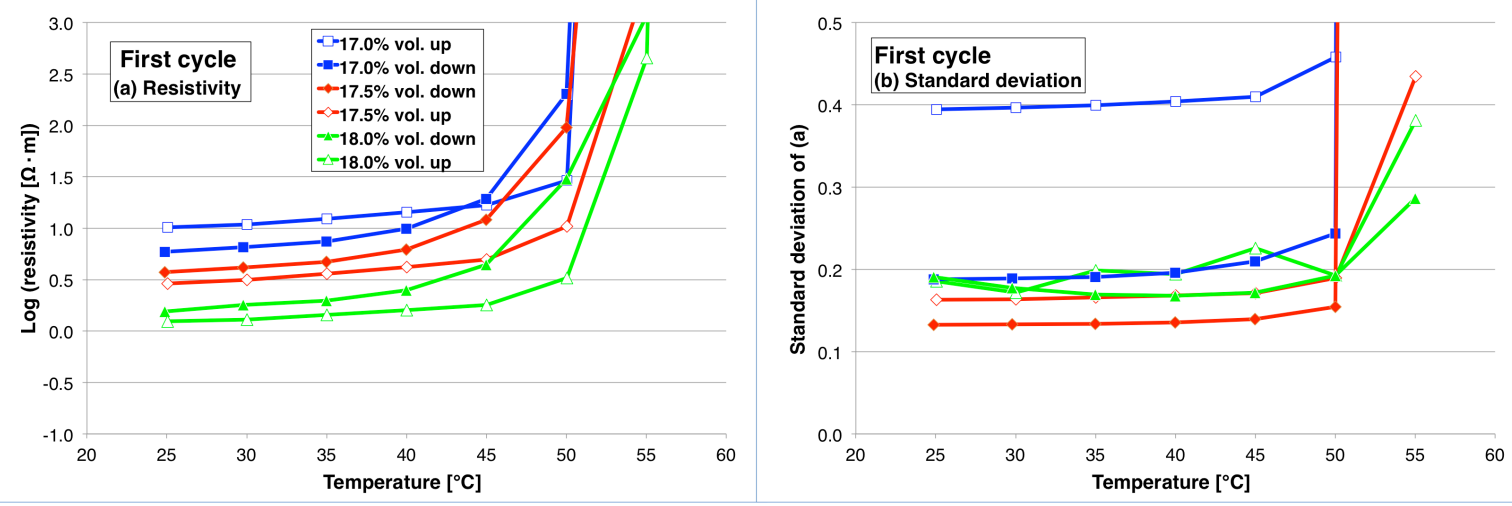

Figure 2. Resistivity for first heating/cooling cycle, all samples.
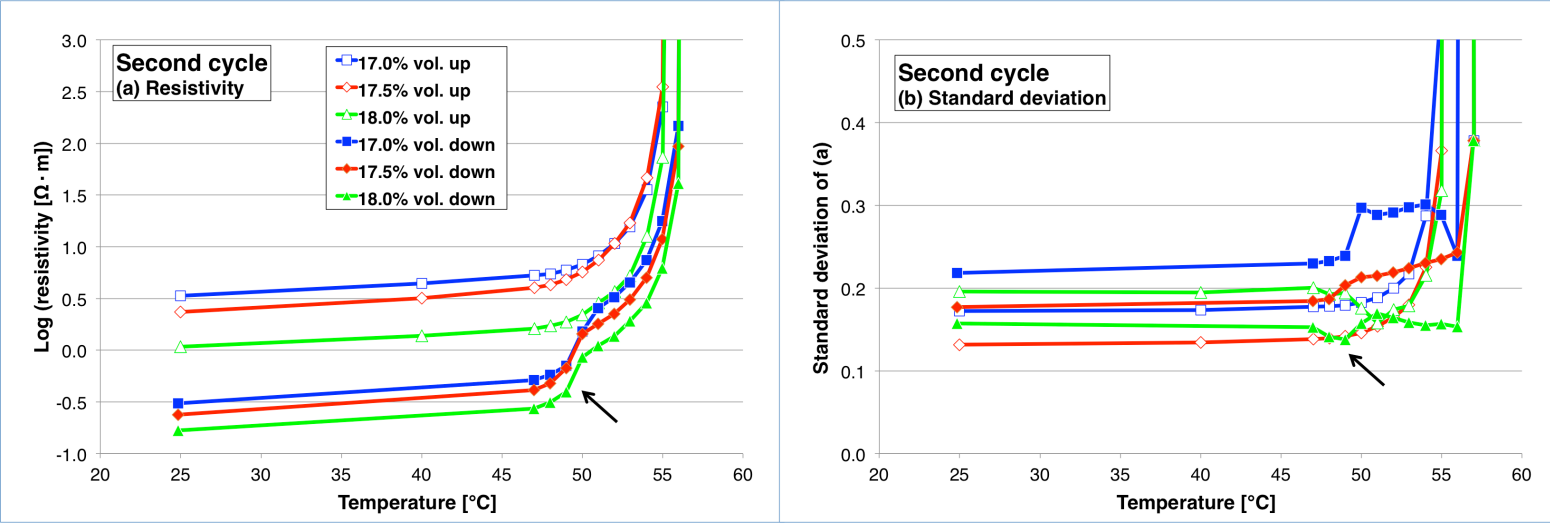

Figure 3. Resistivity for second heating/cooling cycle, all samples. Note additional observable transition upon cooling, indicated by arrow.

Upon slow cooling in the second cycle, an additional observable transition in the resistivity occurs around $50^{\circ} \mathrm{C}$, highlighted by an arrow in Fig. 3. It is tempting to interpret this as the effect of the polymorphic 'rotator'-'crystal' phase transition of common in linear alkanes and 1-alkanols (Al-Mamun, 1974; Kolp and Lutton, 1951; Sirota and Wu, 1996; Tanaka et al., $1959)$, given the good correlation with the corresponding transformation temperature $\left(52^{\circ} \mathrm{C}\right.$ for dry 1-octadecanol according to Sirota and $\mathrm{Wu}$ ). However, although it is reasonable to expect an effect on conductivity through a slight change in volume or a transition in the crystallisation behaviour, the exact mechanism remains to be determined.

Another aspect that further complicates matters is the effect of hydration, which was not controlled in this study: some water may be dissolved in the higher alcohols, and this both increases the melting point and decreases the polymorphic transformation temperature, according to Sirota and $\mathrm{Wu}$, and explains the slight variance in reported transformation temperatures in earlier work with uncontrolled humidity content. Additionally (Al-Mamun, 1974), impurities of neighbouring 1-alkanols are soluble and do not affect the melting point strongly, but depress the polymorphic transformation temperature.

\subsection{Effect of temperature change rate}

Comparing both cycles, it is apparent that the cooling rate considerably influences the resistivity. Although the cooling is in effect stepwise and not strictly continuous, one can nonetheless give average cooling rates around $50^{\circ} \mathrm{C}$, the temperature of the feature seen in the 
$2^{\text {nd }}$ cycle: $30 \mathrm{~K} / \mathrm{h}$ for the $1^{\text {st }}$ cycle, and $9 \mathrm{~K} / \mathrm{h}$ for the $2^{\text {nd }}$. For the first cycle, the resistivity is initially high (after fabrication, i.e. uncontrolled, fast cooling), and remains at roughly the same value after this relatively fast cycle. On the other hand, slower cooling through the crystallisation range of the wax results in much lower resistivity at the end of the cycle, which is seen as both a lower resistivity after the first crystallisation of the wax, and a further decrease from the aforementioned additional transition at $\sim 50^{\circ} \mathrm{C}$. Apparently, the slower cooling gives more time for ordered crystallisation, resulting in a lower resistivity, a trend in line with the increase of the viscosity of the matrix upon cooling (see following section).

Upon further cooling and wax crystallisation, the evolution of the sample is expected to slow down strongly at some point, as both phenomena contribute to crossing of the glass transition of the EC phase, in analogy with the results of Tager for the nitrocellulose-triacetin system (Tager, 1990). Nevertheless, comparing the end of the $1^{\text {st }}$ cycle with the beginning of the $2^{\text {nd }}$ : in the 16-day period between both measurements, resistivity noticeably decreased, indicating that the system is indeed out of equilibrium at room temperature, presumably due to supersaturation of $\mathrm{C} 18 \mathrm{E} 0$ in $\mathrm{EC}$, with further slow crystallisation or rearrangements happening and progressively slowing down as the matrix hardens through corresponding plasticiser loss.

\subsection{Matrix viscosity}

The measurement of the viscosity of the EC:C18E0 matrix on cooling are given in Fig. 4. The crystallisation of the wax is clearly seen (viscosity jump of $>4 \times$ from 49 to $48^{\circ} \mathrm{C}$ ), albeit at a lower transition temperature than the one observed in electrical measurements. We attribute this issue to problems in measuring the true temperature of the sheared liquid with our apparatus, due to 1) the sensor being placed in the tested liquid, but above the strongly sheared volume, 2) conductive and convective heat losses at the top, due to the impossibility with the current setup to immerse this tested volume sufficiently deep in the water mantle, 3) the expected low thermal conductivity of the tested liquid, 4) its high viscosity, precluding significant natural convection. This discrepancy was also visually evident, with surface crystallisation apparent at temperatures clearly above the viscosity transition, nearer to the point indicated by electrical measurements.

Below the initial crystallisation step, viscosity rises roughly exponentially with decreasing temperature, with the fit yielding a $\times 10$ viscosity increase for $\sim 4.7 \mathrm{~K}$ temperature drop. This evolution is expected from three causes: 1) the increase in the volume fraction of the solid, crystalline wax $\mathrm{C} 18 \mathrm{E} 0$ phase, 2) the corresponding decrease of the amount of $\mathrm{C} 18 \mathrm{E} 0$ in the liquid phase, where it plays the role of a solvent / plasticiser, and 3) the temperature effect on the viscosity of the liquid phase. 


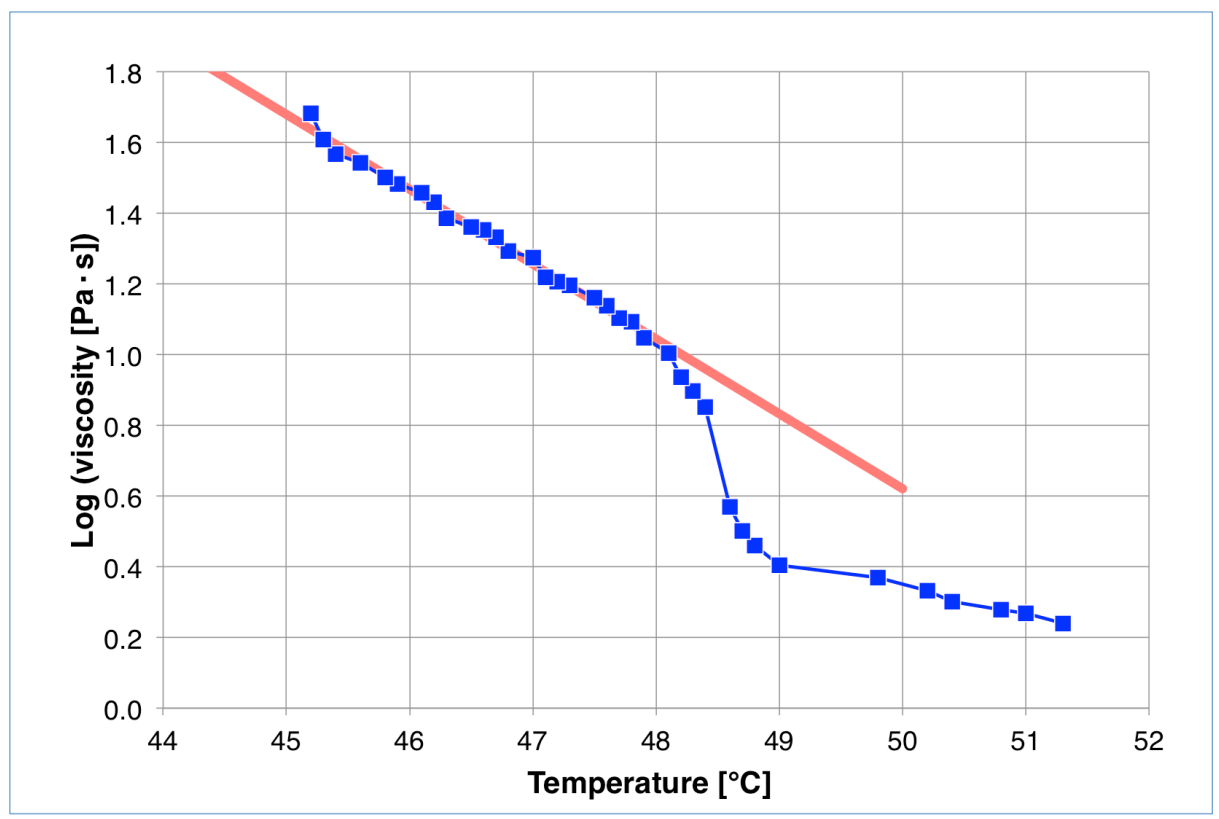

Figure 4. Viscosity of the matrix vs. temperature, at a shear rate of $10 \mathrm{~s}^{-1}$. Linear fit for measurements below $48^{\circ} \mathrm{C}$.

\section{Conclusions and outlook}

The resistivity of composites consisting of a mixed polymer-wax EC-C18E0 matrix, filled with a graphite conductive phase, exhibits 'switching' PTC characteristics slightly below the melting point of the wax, within a certain range of filler concentrations, brought about by the phase behaviour of the polymer-wax system. The results are very promising, as the system may be tuned simply by changing the type and amount of wax, keeping the same base polymer.

However, the results also highlight the complex behaviour of these composites, and further studies are needed to elucidate their equilibrium and kinetic characteristics. In the system formed by EC (or similar polymers) and the higher 1-alkanols, an additional complexity lies in the polymorphic phase behaviour of the alkanols. This question may be answered by using mixtures of near alkanols, which strongly depresses the polymorphic transformation temperature (Al-Mamun, 1974). Another issue lies in the tendency of the polymer phase to "get stuck" at the glass transition, through both cooling and crystallisation of the wax, which acts as a plasticizer in the polymer (Tager, 1990). This issue may also be addressed, by adding another non-crystallisable plasticiser to selectively and controllably depress the glass transition of the polymer phase.

More fundamentally, the nature of the PTC transition is also open to question. A volume effect may play a role, through an increase of the overall matrix density upon crystallisation, resulting in a corresponding increase in the filler volume faction. Such volume effects have indeed been observed in some samples (Zhang, 2005). However, there are some results, for instance on suspensions of exfoliated graphite in water/ice (Zheng, 2011), that clearly require other mechanisms: this system also exhibits a PTC effect, although ice contracts on melting. Therefore, rearrangements involving dispersion / segregation, brought about by melting and crystallisation, are thought to be, in many cases, the dominant mechanism behind the PTC effect (Zhang, 2005). The effect of these postulated mechanisms should be checked in our systems as well, which is made easier by their straightforward tunability. 


\section{Acknowledgements}

The authors acknowledge samples of KS4 graphite from TIMCAL (Bodio, Switzerland), as well as initial experiments with the system from former PhD. student Nathalie Serra.

\section{References}

Al-Mamun, M.A. (1974), "Studies of binary systems of long chain alcohols", Journal of the American Oil Chemists' Society Vol. 51, Iss. 5, pp. 234-237.

Chen, G., Lu, J. and Wu, D. (2005), "PTC effect of polyethylene / foliated graphite nanocomposites", Journal of Materials Science, Vol. 40, pp. 5041-5043.

Chung, D.D.L. (2004), "Electrical applications of carbon materials", Journal of Materials Science, Vol. 39, Iss.8, pp. 2645 2661.

Doljack, F.A. (1981), "PolySwitch PTC devices - a new low-resistance conductive polymer-based PTC device for overcurrent protection", IEEE Transactions on Components, Hybrids, and Manufacturing Technology, Vol. CHMT-4, pp. 372-378.

Domańska, U. and Gonzalez, J.A. (1997), "Solid-liquid equilibria for systems containing long-chain 1-alkanols III. Experimental data for 1-tetradecanol, 1-hexadecanol, 1-octadecanol or 1-icosanol + 1-butanol, 1-hexanol, 1-octanol or 1decanol mixtures. Characterization in terms of DISQUAC", Fluid Phase Equilibria, Vol. 129, No. 1, pp. 139-163.

Dziedzic, A., Czarczynska, H., Licznerski, B.W. and Rangelov I. (1993), "Further examinations of carbon/polyesterimide thick-film resistors", Journal of Materials Science: Materials in Electronics, Vol. 4, pp. 233-240.

Heaney, M.B. (1996), "Resistance-expansion-temperature behavior of a disordered conductor-insulator composite", Applied Physics Letters, Vol. 69, pp. 2602-2604.

Hercules Inc. (now Ashland Inc.), USA (2002), "AQUALON® Ethylcellulose (EC) - physical and chemical properties", product information No. 250-42A 3-02.

Hoerr, C.W., Harwood, H.J., Ralston, A.W. (1944), "Solubilities of high molecular weight normal aliphatic primary alcohols", Journal of Organic Chemistry, Vol. 9, No. 3, pp. 267-280.

Kolp, D.G. and Lutton, E.S. (1951), "The polymorphism of n-hexadecanol and n-octadecanol", Journal of the American Chemical Society Vol. 73, Iss. 12, pp. 5593-5595, 1951.

Łukasik, A., Nowak, S. and Siwulski, S. (2003), "Carbon-polymer posistors heating layer on enamelled steel substrates", in Proceedings, XXVII Int. Conf. IMAPS Poland Chapter, Podlesice, Poland, pp. 207-210.

Maeder, T., Jiang, B., Vecchio, F., Jacq, C., Ryser, P. and Muralt P. (2012), "Lamination of LTCC at low pressure and moderate temperature using screen-printed adhesives", in Proceedings 8th Int. Conf. on Ceramic Interconnect and Ceramic Microsystems Technologies (CICMT), Erfurt, Germany, 2012, pp. 348-352.

Maeder, T., Mietta, J.L., Ruiz, M.M., Jacq, C., Jorge, G., Ryser, P. and Negri, R.M. (2013), "Magnetically tuneable thermoplastic resistive composites", in Proceedings, Electronic Devices and Systems (EDS), IMAPS-CS International Conference, Brno, Czech Republic, 2013, pp. XI-XVIII.

Prudenziati, M. and Dell'Acqua, R. (1994), "Thick film resistors", in Prudenziati, M. (Ed.), Handbook of Sensors and Actuators vol. 1: Thick film sensors, Elsevier, New York, pp. 85-97.

Serra, N., Maeder, T., Lemaire, P. and Ryser P. (2010), "Formulation of composite resistive pastes for fabrication of microheaters", Sensors and Actuators A, Vol. 162, 367-372.

Sirota, E.B. and Wu, X.Z. (1996), "The rotator phases of neat and hydrated 1-alcohols", Journal of Chemical Physics Vol. 105, Iss. 17, pp. 7763-7773.

Strümpler, R. and Glatz-Reichenbach, J. (1999), "Conducting polymer composites", Journal of Electroceramics, Vol. 3, pp. 329-346.

Tager, A.A. (1990), "Solubility of crystalline substances in polymers", Polymer Science U.S.S.R., Vol. 32, No. 10, pp. 19391941.

Tanaka, K., Seto, T., Watanabe A. and Hayashida, T. (1959), "Phase transformation of n-higher alcohols (II)", Bulletin of the Institute for Chemical Research, Kyoto University, Japan, Vol. 37, pp. 281-293.

Zhang, C., Ma, C.A., Wang, P. and Sumita M. (2005), "Temperature dependence of electrical resistivity for carbon black filled ultra-high molecular weight polyethylene composites prepared by hot compaction", Carbon, Vol. 43, pp. $2544-2553$.

Zheng, R., Gao, J., Wang, J. and Chen, G. (2011), "Reversible temperature regulation of electrical and thermal conductivity using liquid-solid phase transitions", Nature Communications, Vol. 2, \#289. 